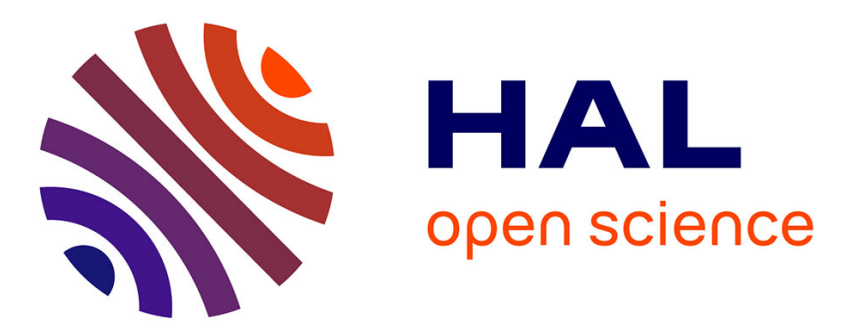

\title{
Double-diffusive sedimentation at high Schmidt numbers: Semi-Lagrangian simulations
}

Jean-Baptiste Keck, Georges-Henri Cottet, Eckart Meiburg, Iraj Mortazavi, Christophe Picard

\section{- To cite this version:}

Jean-Baptiste Keck, Georges-Henri Cottet, Eckart Meiburg, Iraj Mortazavi, Christophe Picard. Double-diffusive sedimentation at high Schmidt numbers: Semi-Lagrangian simulations. Physical Review Fluids, 2021, 6 (2), pp.L022301. 10.1103/PhysRevFluids.6.L022301 . hal-02982080v2

\section{HAL Id: hal-02982080 https://hal.science/hal-02982080v2}

Submitted on 18 Jan 2021

HAL is a multi-disciplinary open access archive for the deposit and dissemination of scientific research documents, whether they are published or not. The documents may come from teaching and research institutions in France or abroad, or from public or private research centers.
L'archive ouverte pluridisciplinaire HAL, est destinée au dépôt et à la diffusion de documents scientifiques de niveau recherche, publiés ou non, émanant des établissements d'enseignement et de recherche français ou étrangers, des laboratoires publics ou privés. 


\title{
Double-diffusive sedimentation at high Schmidt numbers: Semi-Lagrangian simulations
}

\author{
Jean-Baptiste Keck \\ Laboratoire Jean Kuntzmann, \\ Université Grenobles Alpes, \\ 38000 Grenoble, France \\ Georges-Henri Cottet \\ Laboratoire Jean Kuntzmann, \\ Université Grenobles Alpes and CNRS, \\ 38000 Grenoble, France \\ Eckart Meiburg* \\ Department of Mechanical Engineering, \\ University of California, Santa Barbara \\ CA 93106, USA \\ Iraj Mortazavi \\ Laboratoire M2N, EPN6 \\ Conservatoire National des Arts et Métiers \\ 2 rue Conté, 75003 Paris, France \\ Christophe Picard \\ Laboratoire Jean Kuntzmann, Grenoble INP, \\ Université Grenobles Alpes and CNRS, \\ 38000 Grenoble, France ${ }^{\dagger}$
}

(Dated: January 6, 2021) 


\begin{abstract}
When particle-laden freshwater is placed above clear saltwater, double-diffusive sedimentation can arise. Navier-Stokes direct numerical simulations by Burns and Meiburg showed that this process can be dominated by either Rayleigh-Taylor or double-diffusive fingering instabilities. Based on twodimensional simulations, those authors identify a single dimensionless parameter that can be employed to distinguish between these regimes. Here we develop a high-performance semi-Lagrangian computational approach that enables us to extend these high Schmidt number simulations to three dimensions, and to confirm the validity of their proposed scaling law for three-dimensional flows.
\end{abstract}

\title{
I. INTRODUCTION
}

When a less dense layer of warm and salty water is placed above a denser layer of cold and less salty water, the unstable stratification of the more slowly diffusing salt can trigger double-diffusive fingering instabilities [1,2]. An interesting variation of this problem concerns the situation when the more slowly diffusing scalar has a settling velocity associated with it, such as when particle-laden freshwater overlies clear saltwater. These conditions may give rise to double-diffusive sedimentation [3-10], which can strongly alter the effective settling rate of the sediment. This situation was addressed in the linear stability analyses by $[11,12]$ and in the subsequent fully nonlinear simulations by the same groups of authors $[13,14]$. For constant gradient base states, $[15,16]$ furthermore show that the presence of the settling velocity can trigger an entirely new instability mode along with a secondary layering instability, an issue that was addressed in further detail by the recent investigation of [17].

Burns and Meiburg [14] use three-dimensional Navier-Stokes simulations at a diffusivity ratio of 25 to demonstrate the emergence of an interfacial 'nose region' containing both salt and sediment, and whose density can exceed that of the clear salt water below, cf. fig. 1. Hence, there is the potential for Rayleigh-Taylor instabilities to evolve at the lower boundary of the nose region. Depending on the ratio of the nose height $H$ and the effective salinity interface thickness $l_{s}$, the authors argue that the flow is dominated by double-diffusive fingering when $H / l_{s}<O(0.1)$, and by Rayleigh-Taylor instabilities when $H / l_{s}>O(0.1)$.

*meiburg@engineering.ucsb.edu

$\dagger$ Grenoble INP - Institute of Engineering Univ. Grenoble Alpes 

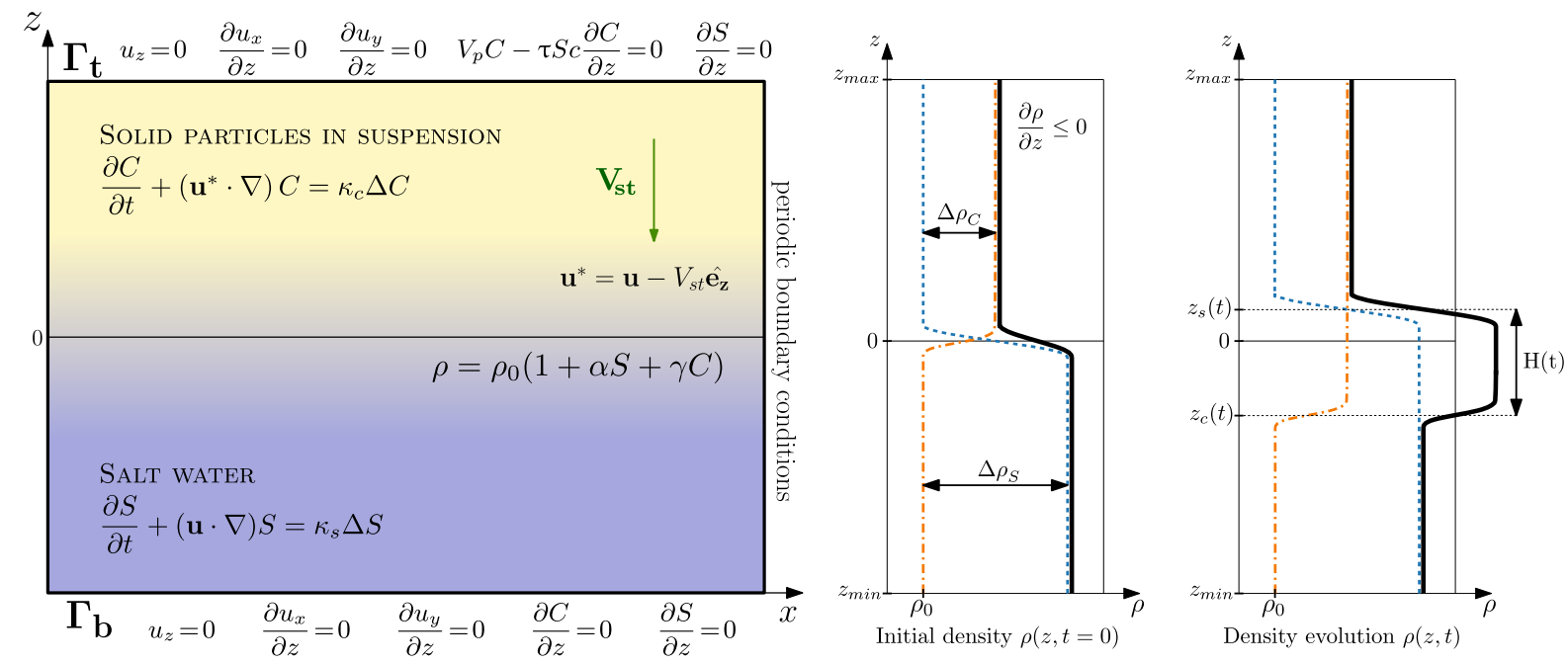

FIG. 1: Particle-laden freshwater above clear saltwater: (a) Problem setup, governing equations and boundary conditions; (b) Sketch of density profiles showing the particle (dash-dotted line), salinity (dashed line) and total density (solid line). The particle settling velocity can result in the formation of a nose region with a maximum total density.

Unfortunately, numerical resolution requirements limited these three-dimensional simulations to unphysically low values of the Schmidt number $S c=\nu_{0} / \kappa_{s}$, which reflects the ratio of the kinematic viscosity $\nu_{0}$ to the diffusivity of salt $\kappa_{s}$. The three-dimensional simulations of [14] employed values of $S c=0.7$ and 7, respectively, as opposed to a realistic value of 700 for salt in water. To access a larger but still somewhat low Schmidt number of 70, the authors resorted to two-dimensional simulations. Results from these ensemble-averaged simulations suggest that the ratio $H / l_{s}$ is a linear function of a single dimensionless grouping that can be interpreted as the ratio of the rates at which sediment flows into and out of the nose region. However, because the proposed scaling law was obtained on the basis of two-dimensional simulations, its validity for three-dimensional flows at realistic Schmidt numbers remains an open question.

To address this issue, the present investigation develops a novel computational strategy based on a semi-Lagrangian approach that allows for the efficient use of hardwarde accelerators, which enables us to extend the fully three-dimensional simulations to more realistic Schmidt number values. After a brief review of the physical problem, we will introduce the semi-Lagrangian computational approach based on the vorticity-velocity formulation of 
the flow equations. The implementation of this approach on hardware accelerators such as GPUs (Graphics Processing Units) will be discussed, along with performance data that demonstrate its ability to simulate realistic double-diffusive flows at very moderate cost. Subsequently, we will focus on how the physics of the three-dimensional double-diffusive sedimentation process changes as we increase the Schmidt number.

The results demonstrate that the scaling law proposed by [14] based on two-dimensional simulations also hold for three-dimensional flows, so that a single dimensionless parameter can predict whether a given flow will be dominated by double-diffusive fingering or by Rayleigh-Taylor instabilities.

\section{PHYSICAL PROBLEM}

The problem set-up is identical to that of Burns and Meiburg [14], in that we consider a less dense layer of particle-laden fresh water above a clear, denser later of salt water, as shown in fig. 1. Here $x$ and $y$ refer to the horizontal directions, whereas $z$ indicates the vertical coordinate. Initially, the interface between the two layers is located at $z=0$.

We assume that the overall density $\rho(\boldsymbol{x}, t)$ depends linearly on salinity $S$ and sediment concentration $C$, with associated density expansion coefficients $\alpha$ and $\gamma$, respectively. The sediment consists of small monodisperse particles with negligible inertia, which are convected by the superposition of the fluid velocity $\boldsymbol{u}(\boldsymbol{x}, t)$ and the Stokes settling velocity $V_{s t}$. The current investigation aims to explore situations where the effective sediment diffusivity $\kappa_{c}$ is substantially smaller than that of the salinity $\kappa_{s}$. We invoke the Boussinesq approximation, and consequently obtain the set of governing equations as

$$
\begin{gathered}
\rho=\rho_{0}+\rho^{\prime}=\rho_{0}(1+\alpha S+\gamma C), \\
\nabla \cdot \boldsymbol{u}=0, \\
\rho_{0}\left[\frac{\partial \boldsymbol{u}}{\partial t}+(\boldsymbol{u} \cdot \nabla) \boldsymbol{u}\right]=\nu_{0} \Delta \boldsymbol{u}-\nabla P+\rho \boldsymbol{g}, \\
\frac{\partial S}{\partial t}+(\boldsymbol{u} \cdot \nabla) S=\kappa_{s} \Delta S, \\
\frac{\partial C}{\partial t}+(\boldsymbol{u} \cdot \nabla) C-V_{s t} \frac{\partial C}{\partial z}=\kappa_{c} \Delta C .
\end{gathered}
$$

Here, $\rho_{0}$ is a constant that indicates the density of clear freshwater and $t$ represents time. 
We follow the approach of Burns and Meiburg [14] to render the above equations nondimensional. By taking the curl of the momentum equation, we thus obtain the set of governing dimensionless equations in the form

$$
\begin{gathered}
\rho^{\prime}=R_{s} S+C, \\
\boldsymbol{\omega}=\nabla \times \boldsymbol{u}, \\
\nabla \cdot \boldsymbol{u}=0, \\
\frac{\partial \boldsymbol{\omega}}{\partial t}+(\boldsymbol{u} \cdot \nabla) \boldsymbol{\omega}=\Delta \boldsymbol{\omega}+(\boldsymbol{\omega} \cdot \nabla) \boldsymbol{u}-\nabla \times \rho^{\prime} \hat{\boldsymbol{e}_{\boldsymbol{z}}}, \\
\frac{\partial S}{\partial t}+(\boldsymbol{u} \cdot \nabla) S=\frac{1}{S c} \Delta S, \\
\frac{\partial C}{\partial t}+(\boldsymbol{u} \cdot \nabla) C-V_{p} \frac{\partial C}{\partial z}=\frac{1}{\tau S c} \Delta C,
\end{gathered}
$$

where $\boldsymbol{\omega}(\boldsymbol{x}, t)$ denotes the vorticity.

The governing dimensionless parameters are given as

\begin{tabular}{rlll}
\hline \hline & Dimensionless parameters & Terminology \\
\hline$R_{s}$ & $=$ & $\alpha S_{\max } / \gamma C_{\max }$ & Stability ratio \\
$\tau$ & $=$ & $\kappa_{s} / \kappa_{c}$ & Diffusivity ratio \\
$S c$ & $\nu_{0} / \kappa_{s}$ & Schmidt number \\
$V_{p}$ & $=$ & $V_{s t} /\left(\nu_{0} g^{\prime}\right)^{\frac{1}{3}}$ & Dimensionless settling velocity \\
$g^{\prime}$ & $\gamma C_{\max } g$ & Reduced gravity \\
\hline \hline
\end{tabular}

We consider a three-dimensional computational domain of size $\Omega=[0,128] \times[0,128] \times$ $[-128,64]$ in the $x$-, $y$ - and $z$-directions. Periodic boundary conditions are applied in the horizontal directions, along with slip walls at the top and bottom boundaries, $\Gamma_{t}$ and $\Gamma_{b}$. We impose vanishing wall-normal derivatives of the salinity at those walls, along with a noflux condition for the sediment concentration at the top wall and a vanishing wall-normal derivative at the bottom wall. We remark that the simulations always terminate before any sediment plumes encounter the bottom wall.

The flow is at rest initially, with salinity and sediment concentration fields in the form of smooth error function profiles as shown on horizontally averaged densities in fig. 1 . 
Initial fields are given as

$$
\begin{gathered}
\boldsymbol{u}_{0}(x, y, z)=\mathbf{0}, \\
\boldsymbol{\omega}_{0}(x, y, z)=\mathbf{0}, \\
C_{0}(x, y, z)=\frac{1}{2}\left[1+\operatorname{erf}\left(\frac{z-\delta(x, y)}{l_{0}}\right)\right], \\
S_{0}(x, y, z)=1-C_{0}(x, y, z) .
\end{gathered}
$$

We choose the initial profile thickness as $l_{0}=1.5$, which represents less than one per cent of the total domain height. A small horizontal random perturbation $\delta(x, y)$ of amplitude $\pm l_{0} / 20$ is imposed on the interface location, in order to trigger the growth of unstable modes. Only stability ratios $R_{s}>1$ are considered, so that the system is initially gravitationally stable.

\section{SEMI-LAGRANGIAN COMPUTATIONAL APPROACH}

The computational approach is based on the velocity-vorticity form of the Navier-Stokes equations in the Boussinesq approximation, and a discretization of the advection-diffusion equations for the vorticity, salinity and sediment concentration fields by a semi-Lagrangian particle method, as will be described in the following.

Particle methods for advective systems discretize the advected quantities via Lagrangian computational elements, referred to as 'particles' (not to be confused with the sediment particles) that move with their respective local velocities [18]. The use of particle methods for simulating variable-density flows dates back to at least [19], who considered the case of a vortex sheet coinciding with a density jump. To handle general flows while ensuring accuracy at all times, the Lagrangian particles can be remeshed on a regular grid via interpolation kernels with strong conservation properties [20]. By remeshing at each time step, we obtain a forward, conservative, semi-Lagrangian method [21, 22]. These methods were applied to various flows including porous media [23, 24]. In the present investigation we employ particles to solve equations (2d), (2e) and (2f), where the quantities carried by the particles are $\boldsymbol{\omega}, S$ and $C$. If $\mathcal{V}=(\boldsymbol{\omega}, S, C)$, one advective step can be written as

$$
\mathcal{V}_{i}^{n+1}=\sum_{j} \mathcal{V}_{j}^{n} \Lambda\left(\frac{x_{i}-x_{j}^{n+1}}{\Delta x}\right) .
$$


Here the superscripts $n$ and $n+1$ refer to two successive time steps, $x_{j}, \mathcal{V}_{j}$ respectively denote the grid points along with the values of $\mathcal{V}$ at those points, $x_{j}^{n+1}$ is the location at time $n+1$ of the particle initialized at $x_{j}$ at time $n$, and $\Lambda$ denotes the interpolation kernel used to remesh the particles. The regular Cartesian grid with mesh size $\Delta x$ on which the particles are remeshed is also used to compute at the end of each time step the velocity from the vorticity, and to calculate vortex stretching, diffusion and the right hand side of (2d). Following [21], we use a direction-by-direction splitting for the advection of particles and the following one-dimensional formula for the remeshing kernel

$$
\Lambda(x)= \begin{cases}1-\frac{5}{4}|x|^{2}-\frac{35}{12}|x|^{3}+\frac{21}{4}|x|^{4}-\frac{25}{12}|x|^{5} & \text { if } 0 \leq|x|<1 \\ -4+\frac{75}{4}|x|-\frac{245}{8}|x|^{2}+\frac{545}{24}|x|^{3}-\frac{63}{8}|x|^{4}+\frac{25}{24}|x|^{5} & \text { if } 1 \leq|x|<2 \\ 18-\frac{153}{4}|x|+\frac{255}{8}|x|^{2}-\frac{313}{24}|x|^{3}+\frac{21}{8}|x|^{4}-\frac{5}{24}|x|^{5} & \text { if } 2 \leq|x|<3 \\ 0 & \text { if }|x| \geq 3\end{cases}
$$

Lagrangian particle advection is performed by an explicit fourth order Runge-Kutta method combined with trilinear velocity interpolation, while remeshing is carried out with the formula given above. This accounts for the advective part of (2d), (2e) and (2f). The stretching term in the right hand side of equation $(2 \mathrm{~d})$ is discretized by fourth order explicit central finite differences. The diffusion terms present on the right hand sides of equations (2d), (2e) and (2f) are solved through an unconditionally stable Fourier-based spectral method adapted to each individual boundary conditions. A similar spectral solver is used to recover vorticity from velocity at the end of each iteration.

For performance reasons, the directional splitting approach used in the semi-Lagrangian particle method to deal with the advection part of the system is further generalized to all finite-difference based operators for vortex stretching and the buoyancy term. These operators can be readily split directionally, as they do not introduce spatial cross-derivatives. Between the treatments of the individual directions, the data are rearranged in memory so that the next direction to be computed becomes contiguous in memory. Data permutation improves the achieved memory bandwidth of the numerical routines and is particularly beneficial when implementing numerical methods with low arithmetic intensity [25, 26]. 

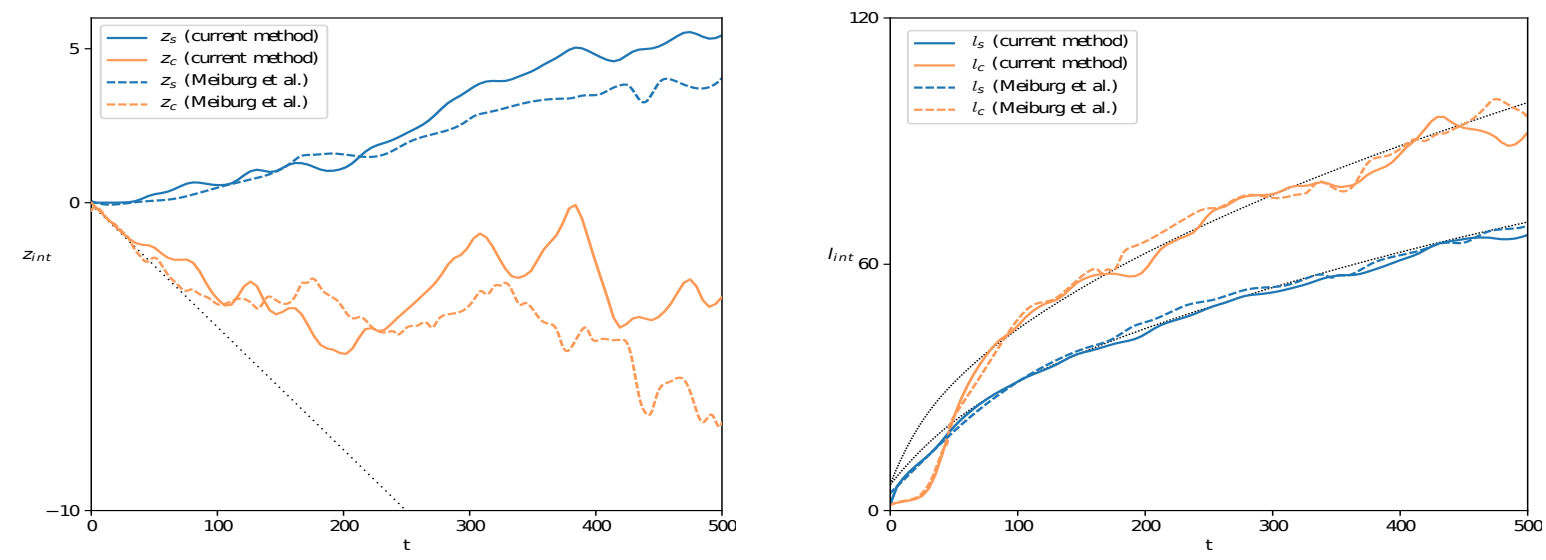

FIG. 2: Comparison of horizontally averaged interface location $z_{\bullet}$ (left) and thickness $l_{\bullet}$ (right), between the present study (solid lines) and the investigation of [14] (dashed lines). Narrow dotted lines represent (a) the location $z=-V_{p} t$ moving with the Stokes settling velocity; and (b) the least-squares fit of the interface thickness to a $\kappa t^{1 / 2}$ diffusive behavior.

\section{IMPLEMENTATION ON HARDWARE ACCELERATORS, PERFORMANCE DATA AND VALIDATION}

GPU implementations of vortex particle methods for 2D flow simulations were first proposed in [27-29]. The present numerical method was implemented within a Python-based library dedicated to 3D flow simulations with semi-Lagrangian particle methods, on various types of accelerators such as GPUs [30]. Within this library, an iteration of the solver is described as a sequence of discretized mathematical operators that are solved successively. Given a single compute node, each operator can run either on the CPU or on an available coprocessor, so that multiple operators can run concurrently in a hybrid CPU-GPU fashion. This sequence of operators is obtained from the topological sort of a direct acyclic graph of operators described by the user [31]. Here we use a single GPU configuration so that each iteration of the solver is driven by the CPU, while the computations take place on the GPU by following the OpenCL standard [32]. All variables such as velocity $\boldsymbol{u}$, vorticity $\boldsymbol{\omega}$, salinity $S$ and sediment concentration $C$ are discretized on Cartesian grids.

Our library deploys an automatic performance-tuning strategy for most operators to ensure performance portability [33]. Spectral operators are implemented by using the clFFT library, which makes use of its own auto-tuning techniques. Nonperiodic boundary conditions are handled via sine and cosine transforms, implemented on top of clFFT by using 
efficient $O(N)$ input pre-processing and output post-processing capabilities of the library.

On a GPU with 32GB of dedicated memory, the above implementation is able to solve single-precision, two-dimensional problems with discretizations up to 32768x16384 (536M grid elements), and three-dimensional configurations up to 1537x512x512 (402M grid elements). Those three-dimensional simulations take $10.9 \mathrm{~s}$ per time step on a single Nvidia Tesla V100 GPU. By comparison, a quad socket Intel Xeon E7-8860 v4 CPU-only platform needs 24 minutes per time step for the same OpenCL implementation coupled to the Intel MKL-FFT library for the spectral transforms and memory mapping. Threedimensional problems addressed below typically require about 1500 time steps, which take around 5 hours in our single GPU configuration.

Semi-Lagrangian particle methods have been validated in [34, 35] for passive scalar transport at high $S c$-values, and in [31] for the classical periodic, incompressible Taylor-Green vortex benchmark at $R e=1,600$. To demonstrate that the solver captures the sedimentation dynamics of interest here, fig. 2 gives a comparison of the flow statistics with results obtained by [14]. In this figure horizontally averaged flow profiles and interface locations and thickness are computed following [14]. Horizontally averaged profiles are computed by integrating each scalar field of interest $\bullet(\boldsymbol{x}, t)$ in the horizontal plane

$$
\langle\bullet\rangle(z, t)=\int_{x_{\min }}^{x_{\max }} \int_{y_{\min }}^{y_{\max }} \bullet(x, y, z, t) \mathrm{d} x \mathrm{~d} y .
$$

We can then track the scalar interfaces for the salinity and sediment concentration fields as follows. Since the error function solves the laminar diffusion equation with constant diffusivity, we compute the least-squares fit of the above horizontally averaged profiles to an error function. For a given time $t$,

1. We fit $\langle C\rangle(z, t)$ against $\langle C\rangle_{f i t}(z, t)=0.5\left[1+\operatorname{erf}\left(\frac{z-z_{c}(t)}{l_{c}(t)}\right)\right]$

2. We fit $\langle S\rangle(z, t)$ against $\langle S\rangle_{f i t}(z, t)=0.5\left[1-\operatorname{erf}\left(\frac{z-z_{s}(t)}{l_{s}(t)}\right)\right]$

This gives $z_{c}, l_{c}, z_{s}$ and $l_{s}$ as functions of time, with the interface position $z_{\bullet}$ defined as the location where $\langle\bullet\rangle_{f i t}=0.5$, and the interface thickness given by $l_{\bullet}$. In our implementation this fit is performed every few time steps and takes as input the last known interface location $z_{\bullet}^{*}$ and thickness $l_{\bullet}^{*}$, with the initial values set to $z_{c}=z_{s}=0$ and $l_{c}=l_{s}=l_{0}$.

Figure 2 indicates that the temporal evolution of these four quantities agrees well with the results of [14]. Further validation results are discussed in [31]. 


\section{RESULTS}

For purely double-diffusive fingering instabilities we would expect to observe a nearly symmetric flow pattern, with slender, low-salinity, clear fluid fingers rising upwards, and corresponding narrow, salty, particle-laden fingers descending from the interfacial region. On the other hand, Rayleigh-Taylor instabilities centered at the density overhang associated with the lower nose boundary should result in strong, downward moving plumes, while any upward propagating plumes should be capped by the stable density stratification at the upper nose boundary. Figure 3 shows the temporal evolution of the $C=0.5$ contour of the sediment concentration field, for $V_{p}=0.04, R_{s}=2$, and $\tau=25$. Results are presented for Schmidt numbers $S c=3.5,7,14$ and 28 (from left to right), and for times $t=100,125$ and 150 (from top to bottom). For the lowest $S c$-value (left column) these figures indeed show slender, up- and downward moving fingers of roughly equal strengths, indicative of a double-diffusive fingering. At the largest value of $S c$ (right column), on the other hand, we observe plumes moving only in the downward direction. The concentration contour exhibits a nearly flat top, where upward plume motion is suppressed by the stable density stratification associated with the upper boundary of the nose region. This confirms the scenario that we had outlined above, in that the flow transitions from being primarily double-diffusive at low $S c$-values to being dominated by Rayleigh-Taylor instabilities at large values of $S c$.

A detailed analysis of these three-dimensional simulation data demonstrates that the horizontally averaged salinity and sediment concentration profiles can be approximated quite well by error function profiles. We take the inflection point of the error function profile as the effective interface location, and the width of the error function profile as the effective interface thickness. In this way, we can evaluate effective interface locations and the thicknesses for salinity and sediment concentration as functions of time. Similar to the two-dimensional simulation profiles in fig. 2, the profiles from the three-dimensional simulations indicate that the center of the salinity interface rises upwards, while that of the sediment concentration descends, so that an effective nose region forms that contains both salt and sediment. The error function fits allow us to evaluate both the nose height $H=z_{s}-z_{c}$ and the thickness $l_{s}$ of the salinity interface as functions of time. While both $H$ and $l_{s}$ individually grow with time, after an initial transient phase the ratio $H / l_{s}$ tends towards a constant value that depends on the governing dimensionless parameters of the flow. 


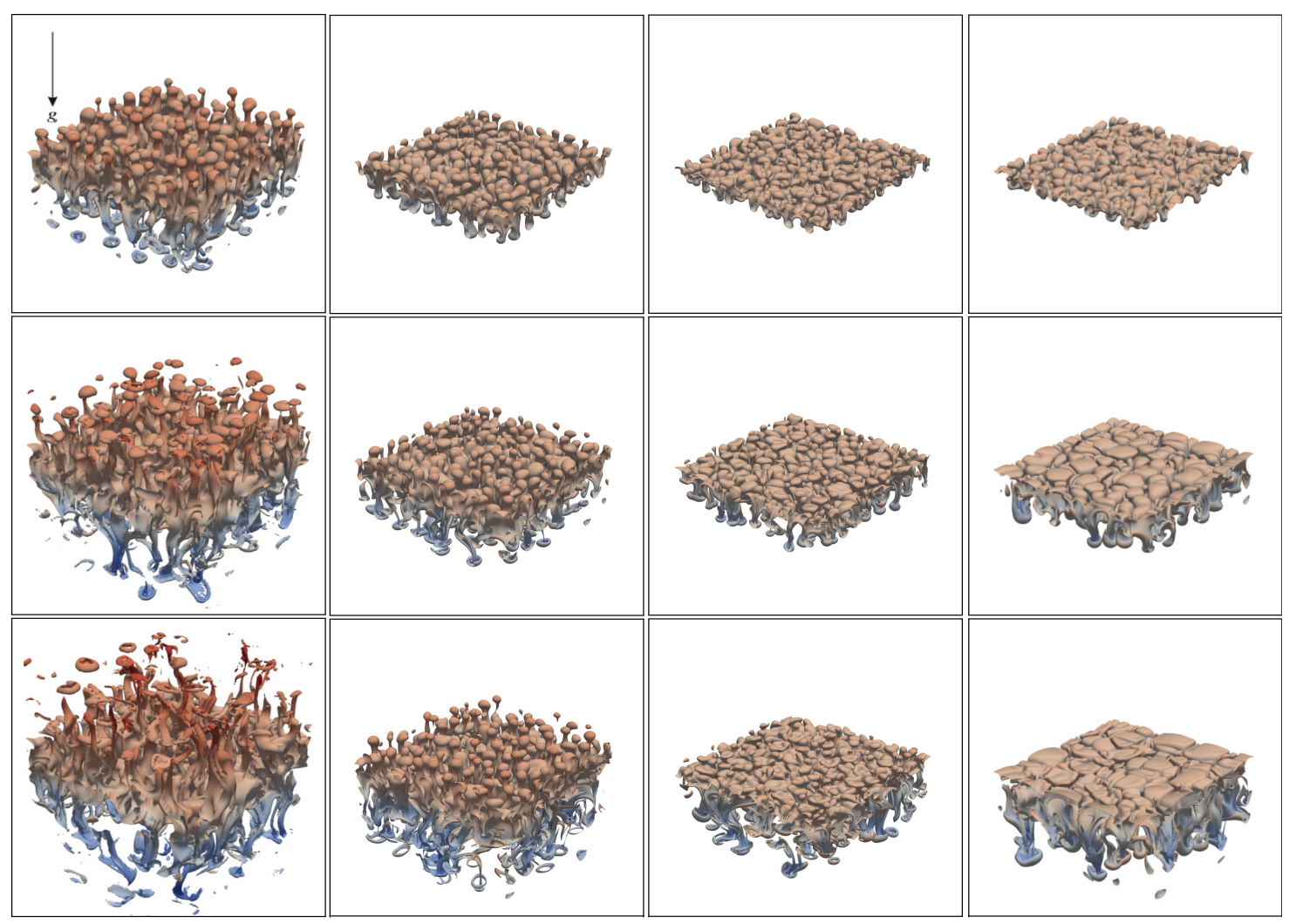

FIG. 3: $C(x, y, z)=0.5$ contours of the sediment concentration, for Schmidt numbers 3.5, 7, 14, 28 (left to right) and times 100, 125, 150 (top to bottom). $V_{p}=0.04, R_{s}=2, \tau=25$.

Fig. 4 compares results for this ratio $H / l_{s}$ of the nose height to the salinity interface thickness, as a function of the dimensionless grouping $V_{p} S c^{1 / 2} / R_{s}$. Data from the present, three-dimensional simulations (plus-signs) are seen to closely match the two-dimensional results of [14]. This confirms that the scaling law proposed by those authors based on two-dimensional simulations remain valid for fully three-dimensional flows.

\section{CONCLUSION}

We have developed a semi-Lagrangian computational approach suitable for being efficiently implemented on hardware accelerators. In this way, we were able to conduct fully resolved three-dimensional simulations of double-diffusive sedimentation at high Schmidt numbers. Consistent with the earlier two-dimensional results of [14], the present threedimensional simulations demonstrate the emergence of an interfacial nose region that contains both salt and sediment. While the height $H$ of the nose region and the thickness $l_{s}$ 


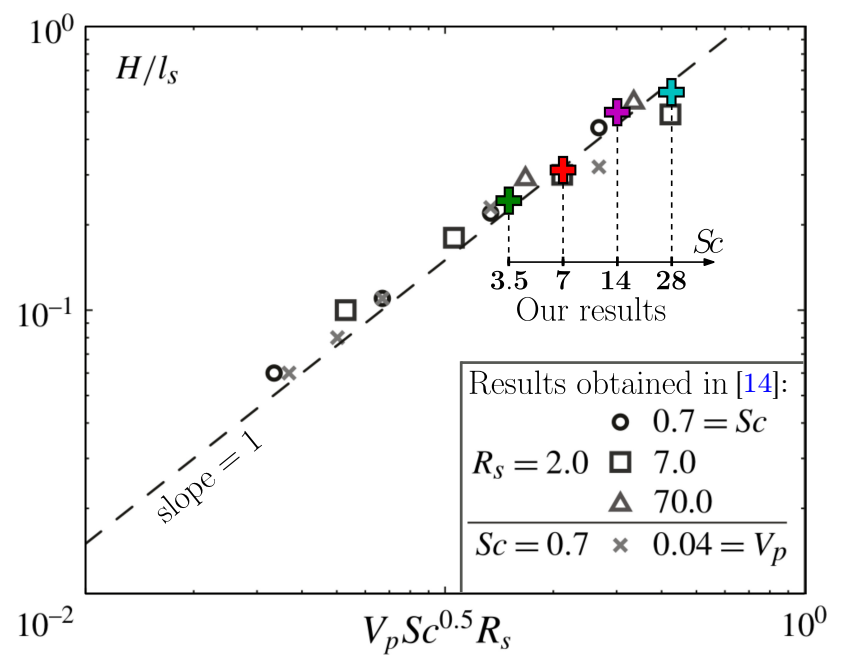

FIG. 4: Ratio $H / l_{s}$ of nose height to salinity interface thickness as function of $V_{p} S c^{1 / 2} / R_{s}$. The two-dimensional results of [14] (o, $\square, \Delta$ and $\boldsymbol{*}$ symbols) closely match the present, three-dimensional results ( $\mathbf{+}$ symbols) for $S c \in\{3.5,7,14,28\}, V_{p}=0.04, R_{s}=2, \tau=25$.

of the salinity interface individually grow with time, the ratio $H / l_{s}$ approaches a constant value that depends only on the governing dimensionless parameters. Depending on whether this ratio is smaller or larger than $O(0.1)$, the sedimentation process is dominated either by double-diffusive fingering or by Rayleigh-Taylor instabilities. The scaling law proposed by [14] for $H / l_{s}$ as function of $V_{p} S c^{1 / 2} / R_{s}$ on the basis of two-dimensional simulations, is seen to remain valid for three-dimensional flows.

As a next step, we plan to implement the semi-Lagrangian computational approach on hardware platforms with multiple GPUs that have recently become widely available. This will allow us to investigate considerably larger systems involving stratified turbulence and double-diffusive phenomena at increasingly realistic Schmidt numbers, in the context of applications in oceanography, limnology and astrophysics.

\section{ACKNOWLEDGMENTS}

The authors would like to thank Peter Burns for providing useful additional numerical implementation details. EM gratefully acknowledges support through NSF grants CBET-1803380 and OCE-1924655, as well as by the Army Research Office through grant W911NF-18-1-0379. JBK and GHC gratefully acknowledge support by Programme Investissement d'Avenir under grant ANR-17-CE23-0024-01. Some of the computations have 
been performed using the GRICAD infrastructure (https://gricad.univ-grenoble-alpes . fr), which is partially supported by grant ANR-10-EQPX-29-01.

JBK performed the numerical computations and data postprocessing. Technical details of the numerical solver were handled by JBK and CP. EM provided most of the physical input and discussed the results. GHC and IM delivered their expertise in semi-Lagrangian methods. GHC, IM and CP supervised the findings of this work. All authors provided critical feedback and contributed to the final manuscript.

\section{REFERENCES}

[1] J. S. Turner, Salt fingers across a density interface, Deep-Sea Res. 14, 599 (1967).

[2] J. S. Turner, Double diffusive phenomena, Annual Review of Fluid Mechanics 6, 37 (1974).

[3] T. Green, The importance of double diffusion to the settling of suspended material, Sedimentology 34, 319 (1987).

[4] C. F. Chen, Particle flux through sediment fingers, Deep-Sea Res. 44, 1645 (1997).

[5] D. C. Hoyal, M. I. Bursik, and J. F. Atkinson, The influence of diffusive convection on sedimentation from buoyant plumes, Marine Geology 159, 205 (1999).

[6] D. C. Hoyal, M. I. Bursik, and J. F. Atkinson, Settling-driven convection: a mechanism of sedimentation from stratified fluids, J. Geophys. Res. 104, 7953 (1999).

[7] T. Maxworthy, The dynamics of sedimenting surface gravity current, Journal of Fluid Mechanics 392, 27 (1999).

[8] J. D. Parsons and M. H. Garcia, Enhanced sediment scavenging due to double-diffusive convection, J. Sedim. Res. 70, 47 (2000).

[9] J. D. Parsons, J. W. M. Bush, and J. P. M. Syvitski, Hyperpycnal plume formation from riverine outflows with small sediment concentrations, Sedimentology 48, 465 (2001).

[10] V. Manville and C. Wilson, Vertical density currents: a review of their potential role in the deposition and interpretation of deep-sea ash layers, J. Geol. Soc. 161, 947 (2004).

[11] P. Burns and E. Meiburg, Sediment-laden fresh water above salt water: linear stability analysis, Journal of Fluid Mechanics 691, 279 (2012).

[12] X. Yu, T.-J. Hsu, and S. Balachandar, Convective instability in sedimentation: Linear stability analysis, Journal of Geophysical Research: Oceans 118, 256 (2013). 
[13] X. Yu, T.-J. Hsu, and S. Balachandar, Convective instability in sedimentation: 3-d numerical study, Journal of Geophysical Research: Oceans 119, 8141 (2014).

[14] P. Burns and E. Meiburg, Sediment-laden fresh water above salt water: nonlinear simulations, Journal of Fluid Mechanics 762, 156 (2015).

[15] A. Alsinan, E. Meiburg, and P. Garaud, A settling-driven instability in two-component, stably stratified fluids, Journal of Fluid Mechanics 816, 243-267 (2017).

[16] J. F. Reali, P. Garaud, A. Alsinan, and E. Meiburg, Layer formation in sedimentary fingering convection, Journal of Fluid Mechanics 816, 268-305 (2017).

[17] R. Ouillon, P. Edel, P. Garaud, and E. Meiburg, Settling-driven large-scale instabilities in double-diffusive convection, Journal of Fluid Mechanics 901, A12 (2020).

[18] P. A. Raviart, An analysis of particle methods, in Numerical Methods in Fluid Dynamics, Lecture Notes in Math., Vol. 1127 (1983).

[19] C. Anderson, A vortex method for flows with slight density varaition, Journal of Computational Physics 61, 417 (1985).

[20] G.-H. Cottet and P. D. Koumoutsakos, Vortex methods: theory and practice (Cambridge university press, 2000).

[21] G.-H. Cottet and A. Magni, TVD remeshing schemes for particle methods, C. R. Acad. Sci. Paris Ser. I, 347, 1367 (2009).

[22] G.-H. Cottet, J.-M. Etancelin, F. Pérignon, and C. Picard, High order semi-lagrangian particle methods for transport equations: numerical analysis and implementation issues, ESAIM: Mathematical Modelling and Numerical Analysis 48, 1029 (2014).

[23] C. Mimeau, G.-H. Cottet, and I. Mortazavi, Direct numerical simulations of three-dimensional flows past obstacles with a Vortex penalization method, Computers and Fluids 136, 331 (2016).

[24] C. Mimeau, I. Mortazavi, and G.-H. Cottet, Passive control of the flow around a hemisphere using porous media, Eur. J. Mech - B/Fluids 65, 213 (2017).

[25] C. Carvalho, The gap between processor and memory speeds, in Proc. of IEEE International Conference on Control and Automation (2002).

[26] G. Ofenbeck, R. Steinmann, V. Caparros, D. G. Spampinato, and M. Püschel, Applying the roofline model, in 2014 IEEE International Symposium on Performance Analysis of Systems and Software (ISPASS) (IEEE, 2014) pp. 76-85.

[27] D. Rossinelli and P. Koumoutsakos, Vortex methods for incompressible flow simulations on the 
gpu, The visual computer 24, 699-708 (2008).

[28] D. Rossinelli, M. Bergdorf, G.-H. Cottet, and P. Koumoutsakos, GPU accelerated simulations of bluff body flows using vortex particle methods, Journal of Computational Physics 229, 3316-3333 (2010).

[29] D. Rossinelli, B. Hejazialhosseini, W. van Rees, M. Gazzola, M. Bergdorf, and P. Koumoutsakos, MRAG-I2D: Multi-resolution adapted grids for remeshed vortex methods on multicore architectures, Journal of Computational Physics 288, 1-18 (2015).

[30] J.-M. Etancelin, G.-H. Cottet, F. Pérignon, and C. Picard, Multi-CPU and multi-GPU hybrid computations of multi-scale scalar transport, in 26th International Conference on Parallel Computational Fluid Dynamics (Trondheim, Norway, 2014) pp. 83-84.

[31] J.-B. Keck, Numerical modelling and High Performance Computing for sediment flows, Phd thesis, Université Grenoble Alpes (2019).

[32] J. Tompson and K. Schlachter, An introduction to the opencl programming model, Person Education 49, 31 (2012).

[33] Y. Zhang, M. Sinclair, and A. A. Chien, Improving performance portability in opencl programs, in International Supercomputing Conference (Springer, 2013) pp. 136-150.

[34] J.-B. Lagaert, G. Balarac, and G.-H. Cottet, Hybrid spectral-particle method for the turbulent transport of a passive scalar, Journal of Computational Physics 260, 127 (2014).

[35] J.-M. Etancelin, Model coupling and hybrid computing for multi-scale CFD, Phd thesis, Université de Grenoble (2014). 